\title{
An Eye Movement Study on the Relationship Between Multiple Implicit Sequence Learning and Attention
}

\author{
Zhanglong $\mathrm{Lu}^{1,}$,, Xiaoyu $\mathrm{Li}^{2}$ \\ ${ }^{1}$ College of Education, Zhengzhou University, Zhengzhou, China \\ ${ }^{2}$ Zhengzhou Central Hospital, Zhengzhou, China
}

Email address:

psylzl@zzu.edu.cn(Zhanglong Lu)

${ }^{*}$ Corresponding author

\section{To cite this article:}

Zhanglong Lu, Xiaoyu Li. An Eye Movement Study on the Relationship Between Multiple Implicit Sequence Learning and Attention. Psychology and Behavioral Sciences. Vol. 7, No. 1, 2018, pp. 8-13. doi: 10.11648/j.pbs.20180701.13

Received: March 2, 2018; Accepted: March 19, 2018; Published: April 16, 2018

\begin{abstract}
The purpose of this study was to explore the relationship between multiple implicit sequence learning and attention. A one-factor between-subjects experimental design was used, with attentional load (low vs. high) as between-subjects variable. Eye-movement technology was adopted, and saccadic reaction time was as dependent measure. Forty healthy volunteers were randomly assigned to high attentional load condition and low attentional load condition. The results showed that: (1) Saccadic reaction time in high attentional load condition was longer than low attentional load condition's; (2) Both the primary sequence and the secondary sequence could be learned no matter whether in low attentional load condition or in high attentional load condition; (3) the sequence learning scores did not differ from primary sequence and secondary sequence. These findings suggest that there are no attentional limitations on the learning of multiple sequence learning.
\end{abstract}

Keywords: Multiple Sequence Learning, Implicit Learning, Attention, Eye Movements

\section{Introduction}

Implicit learning is assumed to be one of the most fundamental learning processes enabling humans to learn regular structures in the complex environment. Humans usually do this without any intention or any effort and do not aware what they actually learn. Serial reaction time (SRT) task paradigm is one of the most used paradigms in the study of implicit learning. Since the serial reaction time (SRT) task paradigm was originated by Nissen and Bullemer [1], a lot of psychologists have engaged in the study of implicit learning [2-10]. Yet, there has no agreement neither on the empirical nor on the theoretical side.

Most of the former studies focused on the single sequence learning, but implicit learning system in humans is sensitive to complex environmental contingencies [11-12], the implicit learning system would allow for the effortless acquisition of powerful cognitive abilities (e.g. language, learning). The automatic nature of implicit learning appeared to be that a primary sequence and secondary sequence can be learned in parallel, where the secondary sequence learning is acquired as byproduct of performing the primary sequence task. Some studies have explored whether two sequences could be learned in parallel.

May [13] obtained evidence that participants could learn both a sequence of object identities (the primary sequence) and a sequence of locations in parallel. Participants responded to an object faster when its identity and the location were predictable, compared to only one dimension being predictable. Jiménez and Méndez [14] demonstrated that a sequence of locations can be learned and not be impaired when participants simultaneously learned a relationship between shapes and locations. Besides Cock, Berry and Buchner [15] also found that two sequences of locations could be learned simultaneously when one sequence was attended and the other sequence was ignored. Röttger, Haider, Zhao and Gaschler [16] asked participants to complete visual SRT task and tone-discrimination task simultaneously, both of two tasks followed a predetermined sequence. The findings revealed that both the SRT sequence and the tone sequence could be learned in parallel as long as there were correlations or fixed 
combinations (across-task predictability) between the SRT sequence and the tone sequence. Sloutsky and Robinson [17] found 1-2 years old young infants can learn multiple contingencies in parallel when there is sufficient redundancy in the input. Sævland and Norman [18] also found participants can complete the Alternating Serial Reaction Time task and Sugar Factory Task.

Particularly Rowland and Shanks [19] was the first study which aimed to test the idea that human learn multiple visual sequence learning simultaneously and the learning may proceed in the absence of attention. Rowland and Shanks used two independent probabilistic sequences to explore whether human could learn two sequence learning simultaneously without attention. Rowland and Shanks found participants could learn a primary sequence and a secondary sequence in parallel when the perceptual load of the primary task was low, However, learning of the secondary sequence was not obtained if the perceptual load of the primary task was high. These results showed attention modulates the learning of multiple sequence learning. Albeit Rowland and Shanks' study has largely contributed to our understanding of multiple sequence learning, one potential weakness should be mentioned. The eye movement was not controlled in Rowland and Shanks' study. The eye movement was a very important factor because the materials used in Rowland and Shanks' study were arranged in two rows. Participants were asked to respond to a primary sequence which was in the bottom row while ignoring a secondary sequence which was in the top row. But, participants may concentrate on the top row during the training stage. So the goal of the present study was to further investigate the relationship between multiple implicit sequence learning and attention. For this purpose, the present study used an experimental setup similar to the paradigm used by Rowland and Shanks. The exceptions were (a) the eye tracking technology was used to control the eye movement during the experiment; (b) taking into account it is not clear whether the complexity of the multiple implicit sequence might have affected the relationship between multiple implicit sequence learning and attention, the deterministic sequences were used instead of probabilistic ones. During the training stage, participants responded to a primary sequence while ignoring a secondary sequence, and during the test stage, participants responded to the primary sequence, the secondary sequence, and a new sequence. The main interest was to investigate whether participants learned more about the secondary sequence in the low attentional load condition than in the high attentional load condition. To assess any difference in the learning scores between the high attentional load condition and the low attentional load condition, the magnitude of learning scores between the two conditions were compared. In short, the goal of the present study was to contribute the debate about the relationship between implicit learning and attention by using eye tracking technology to test multiple implicit sequence learning in low and high attentional load condition. We hypothesized that secondary sequence learning was obtained in the low and high attentional load condition, and there was no significant difference between the low and high attentional load condition.

\section{Materials and Methods}

\subsection{Ethics Statement}

The study was approved by the Ethics Committee of Zhengzhou University. An informed consent was obtained from all participants prior to participation in the experiment.

\subsection{Participants}

Forty students from Zhengzhou university (31 female, 9 male) participated in the study and were given payment. The average of the participants was 20.63 years ( $S D=1.59$ years). All participants had normal or corrected-to-normal vision and had not previously taken part in implicit sequence learning experiment and were unaware of the purpose of the experiment. Participants were randomly assigned to one of two experimental conditions (low attentional load condition, $\mathrm{n}$ $=20$; high attentional load condition, $\mathrm{n}=20$ ).

\subsection{Materials}

The stimuli in the SRT task consisted of eight black squares on a white background, eight black squares were arranged in two rows of four horizontally aligned black squares. A red circle appeared in the bottom and the top row of four locations respectively (referred to as $1-4$, from left to right). Besides in the high attentional load condition, A green circle and a red square appeared in the bottom row as distractor stimuli. Unbeknownst to the participants, in all conditions the successive locations of the target followed a 12-item deterministic sequence ( the primary sequence $=4-1-3-2-1$ $-2-4-3-1-4-2-3$; the secondary sequence= 2-3-2-1-4-3-1-2-4-1-3-4; the new sequence= $3-4-1-2-4$ $3-1-4-2-1-3-2)$. The sequences were balanced for frequency (each location occurred three times), transition frequency (each possible transition from one location to another occurred once), repetitions (no repetitions in either sequence) and rate of full coverage.

\subsection{Experimental Design}

A one-factor between-subjects experimental design was used, with attentional load condition (low vs. high) as between-subjects variable. The dependent variable was the saccadic response time.

\subsection{Apparatus}

An SR Research (Mississauga, Ontario, Canada) EyeLink 1000 plus eye-tracking system recorded subjects' eye movements with a sampling rate of $1,000 \mathrm{~Hz}$. This eye tracker has a high spatial $\left(0.01^{\circ}\right.$ of visual angle) using pupil tracking and corneal reflection, The materials was displayed on a 19-in. Dell monitor with a refresh rate of $75 \mathrm{~Hz}$ (resolution 1,024 $\times 768$ pixels), the viewing distance was approximately $60 \mathrm{~cm}$. Viewing was binocular and only right eye was tracked as permitted by the quality of the calibration for right eye. The 
experiment was run with Experiment Builder 1.10 software.

\subsection{Procedure}

\subsubsection{SRT Task}

All participants were seated in a silent room with normal ambient illumination with the head positioned on a chin rest, and were randomly assigned into low or high attentional load condition In order to make sure that participants had understood the instructions, participants in both high attentional load condition and low attentional load condition started with a practice block which consisted of 50 trials. In all these practice trials, the stimulus did not follow any regular sequence.

After these practice trials, the participants performed 19 blocks of 120 trials for a total of 2280 trials for an SRT task, which comprised 16 blocks of training followed by 3 blocks of testing. In each block, the sequence started at a random position to counterbalance the order of 4 positions, and each session was separated by a short break. In each trial a red circle appeared in the bottom and top row respectively, participants were given instructions to look at the red circle as fast and as accurately as possible, but participants did not know that the red circle would appear in a predetermined sequence. Each trial began with a central fixation cross. The fixation "+" appeared in the center of the screen for $250 \mathrm{~ms}$, then the red circle was presented immediately. During the training phase, participants responded to the location of a red circle (the target stimulus) in the bottom row and ignored the red circle in the top row. Reversely, during the testing phase, participants were instructed to react to the stimulus in the top row and ignore the red circle in the bottom row where the primary sequence remained. For the 17 th block, responding to the target stimulus in the top row, the sequence was the secondary sequence (i.e., the ignored sequence that had appeared in the top row during the learning sessions). And for the 18th block, responding to the target stimulus in the top row, and the sequence was a new sequence. And for the 19th block, responding to the target stimulus in the top row, and the sequence was the primary sequence. In short, we examined learning of the primary and the secondary sequence over a 19-block SRT session.

After participants had read the instruction, participants were calibrated with a standard 5-point grid for both eyes. Calibration was applied at the beginning of every session. The experimenter checked the accuracy of the calibration at any time and recalibrated if necessary during the whole process of experiment, especially participants' eye was not detected by the eye tracker.

\subsubsection{Generation Task}

After the serial reaction time task portion of the experiment was finished, all participants conducted a generation task test [20]. The purpose of the generation task was to assess the extent to which participants could control their sequence knowledge strategically. Evaluating the level of explicit knowledge is important, that is because implicit learning and explicit learning are fundamentally different from each other and even supported by different systems.

Participants were informed that the order of target positions followed a predetermined sequence, in which every location was completely determined by the previous two locations. According to the Process Dissociation Procedure [21], under the inclusion condition participants were asked to generate a sequence that resembled the learning sequence as much as possible. Conversely, under the exclusion condition participants were asked to generate a new sequence, but participants had to try to avoid generating the sequential regularities of the training sequence. In both cases, a restriction was implemented, namely that participants were forbidden from generating the same location twice or more in a row. Half of the participants did the inclusion test firstly, and then the exclusion test; and the other half participants completed the exclusion test firstly, and then the inclusion test.

\section{Results}

All analyses were conducted in Data Viewer version 1.11 and Statistical Product and Service Solutions (SPSS) version 21.0. Data from three participants who did not complete the whole experimental task were removed, leaving $n=37$ participants for the following analysis. Figure 1 shows the mean saccadic response time in high and low attentional load group obtained over the training phase.

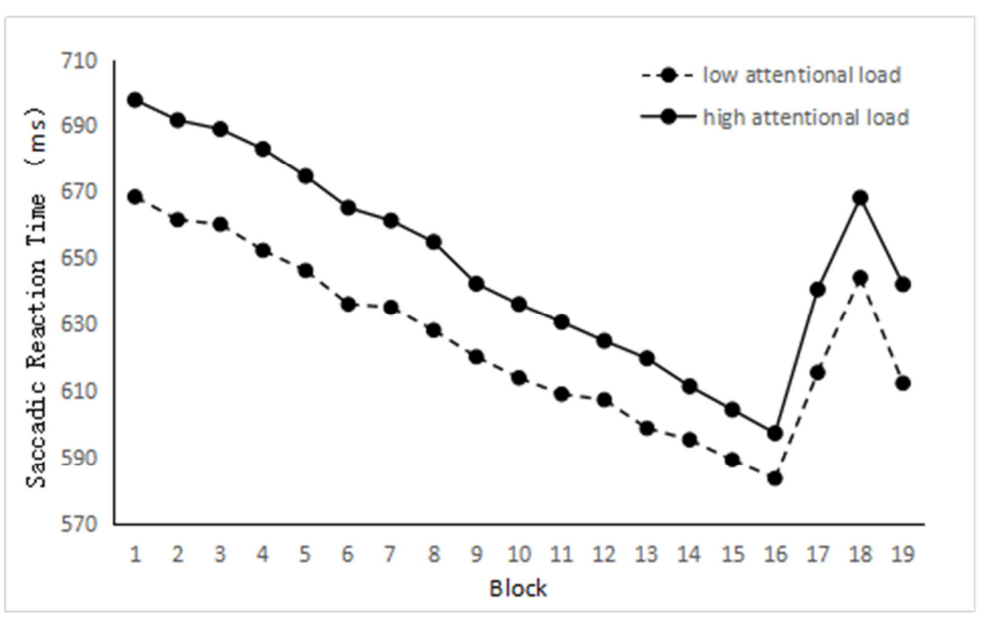

Figure 1. Saccadic reaction time in low and high attentional load. 
As can be seen in Figure 1, saccadic response time decreased gradually from Block 1 to Block 16 in both group. In Block 17, where a secondary sequence was presented, saccadic response time increased in both group. In Block 18, where a new sequence was presented, saccadic response time increased in both group. And saccadic response time decreased again in Block 19 where the primary sequence was resumed. To test this effect statistically, a 2 (Group: low attentional load, high attentional load) $\times 11$ (Block:1-16) mixed-design Analysis of Variance (ANOVA) was carried out with Block as a within-subjects variable and Group as a between-subjects variable. The results showed that the main effect of Block was significant, $F(15,525)=9.01, p<0.001$, $\eta^{2}=0.21$, and saccadic reaction time was less with increasing blocks.

In order to determine whether participants had acquired knowledge of the learning sequence and whether the two groups had acquired the same or different amount of knowledge. The last three blocks (Block 17, Block 18 and Block 19) performance were determined as sequence knowledge. The performance in the learning sequence blocks (average performance of Blocks 17 and 19) with the performance in the Block 18 (a new sequence) were compared. Sequence knowledge should improve participants' performance, if participants had acquired sequence knowledge, the performance should decline when the learning sequence was new in Block 18. To obtain a measure of sequence learning, a 2 (Group: low attentional load, high attentional load) $\times 2$ (Sequence: primary, secondary) mixed-design Analysis of Variance (ANOVA) was carried out with Sequence as a within-subjects variable and Group as a between-subjects variable. The results suggested that there was no significant difference. The learning scores for the primary sequence in the high attentional load condition were $48.40 \mathrm{~ms}(S D=42.80)$, and in the low attentional load condition were $46.17 \mathrm{~ms}(S D=17.09)$. The learning scores for the secondary sequence in the high attentional load condition were $27.54 \mathrm{~ms}(S D=6.37)$, and in the low attentional load condition were $28.67 \mathrm{~ms}(S D=16.39)$.

\section{Discussion}

The current study investigated whether multiple implicit sequence learning requires attention, or if multiple implicit sequence learning can occur without attention. So the difference between the high attentional load condition and the low attentional load condition was assessed by comparing the learning scores. Participants were instructed to look at a target stimulus in the bottom row while ignoring the top row during the training stage; on the contrary, looking at a target stimulus in the top row while ignoring the bottom row during the testing stage. The results demonstrated that multiple implicit sequence learning occurred no matter the attentional load was low or high, and there was no significant difference between low attentional load condition and high attentional load condition.
The results are inconsistent with the previous finding that suggests that multiple implicit sequence learning requires attention [19]. As the former studies focused on implicit sequence learning, even subtle changes in the experimental setups and research designs might have provoked differences in the implicit sequence learning performance. This, in turn, could have contributed to the divergent findings and complicates comparisons across studies. Note that in Rowland and Shanks' [19] paradigm, it was difficult to prohibit participants from concentrating on the secondary sequence during the training stage. Therefore, it was possible that participants learned the secondary sequence when they concentrated on the top row. The current study solved this problem by using eye tracking technology and clearly demonstrated that participants did not concentrate on the top row during the training stage. Higuchi and Saiki [22] have demonstrated that implicit learning occurs in parafoveal vision when eye movement was restricted. Future study can further employ a SRT task display that corrected the size of each item according to its distance from the fixation point [22-23]. This manipulation ensured that each stimulus was equally visible when participants maintain fixation on the center of the displays. Moreover compared with Rowland and Shanks [19], the present study used deterministic sequences rather than probabilistic ones. The type of sequence affects performance of sequence learning [12]. Compared with probabilistic sequence, deterministic sequence was easier to learn. In Rowland and Shanks' study, when the perceptual load of the primary was low, Rowland and Shanks found both the primary and secondary sequence could be learned. But when the perceptual load of the primary was high, the secondary sequence learning disappeared. While in the present study, deterministic sequence might reduce the difficulty of learning, even on the high perceptual load condition, participants could learn the secondary sequence.

One possible explanation for this results is that the primary deterministic sequence and the secondary deterministic sequence are successfully integrated into a deterministic sequence after a large quantity of learning. The results are in line with task integration hypothesis [24]. Schmidtke and Heuer [24] used the tone-counting task and SRT task, Participants pressed a foot-pedal in response to one of the two tones (go/no go task). The tones followed a 6-elements or a 5 -elements sequence. Thus the tones were correlated with the 6-elements SRT sequence to a high or to a low degree. Schmidtke and Heuer found larger amounts of sequence learning with the 6-elements tone sequence than with the 5 -elements tone sequence. Because participants had integrated the 6-elements tone sequence into the SRT sequence resulting in a 12-elements sequence and a more difficult 60 -elements sequence in the latter case. Similarity integrating two 12-elements probabilistic sequences [19] into a sequence was more difficult than two 12-elements deterministic sequences (e.g. the present study) into a sequence. Another possible explanation for this results is Lavie's theory of attention [25]. Lavie proposed attention has both resources and selection 
properties. Selection is effortful and becomes more focused when resources are scarce. Conversely, when the current task is easy, excess resources are available for selecting other irrelevant task. And Lavie distinguished two types of attentional load: perceptual load and working memory or cognitive load. In contrast to the effects of perceptual load, high working memory load appears to increase interference from irrelevant distractors because working memory load reduces the capacity of control functions. This can explain why primary sequence learning was not affected by an increase in high perceptual load, because under the high perceptual load condition, adequate central control ensured reduced interference from distractors. Multiple implicit sequence learning was unaffected by increased perceptual load suggested that multiple implicit learning was highly resistant to noise, or input complexity, multiple implicit learning extracted regularities from the environment and reduced interference from noise. Recently the similar opinion was approved by Schumacher and Schwarb [26]. Schumacher and Schwarb proposed that parallel response selection (central capacity sharing) played a major role in implicit sequence learning. In Schumacher and Schwarb's experiments, participants were instructed to respond manually to the visually presented SRT stimuli and verbally to the tones. The tones were presented with the SRT stimuli simultaneously and not, as was done in most former studies. Schumacher and Schwarb found parallel-interfering central processing disrupts sequence learning, SRT task performance can be disrupted without disrupting sequence learning when that disruption involves a response-selection bottleneck rather than parallel response selection. The results suggest that it is the overlap of central processes involved in implicit sequence learning that leads to learning deficits in sequence learning. Both Lavie and Schumacher et al. emphasized on the importance of central control capacity during the implicit sequence learning. However, Future study is needed to further examine the influence of cognitive load (e.g. working memory) on multiple implicit sequence learning, and more research will be necessary to further examine the mechanism of multiple implicit sequence learning.

\section{Conclusion}

The current study showed that multiple sequence learning could be learned implicitly. Moreover, there were no attentional limitations on the learning of multiple implicit sequence learning.

\section{References}

[1] Nissen, M. J., \& Bullemer, P. (1987). Attentional requirements of learning: Evidence from performance measures. Cognitive Psychology, 19, 1-32.

[2] Deroost, N., Coomans, D., \& Soetens, E. (2009). Perceptual Load Improves the Expression but not Learning of Relevant Sequence Information. Experimental Psychology, 56 (2), 84-91.
[3] Franklin, M. S., Smallwood, J., Zedelius, C. M., Broadway, J. M., \& Schooler, J. W. (2016). Unaware yet reliant on attention: Experience sampling reveals that mind-wandering impedes implicit learning. Psychon Bull Rev, 23: 223-229.

[4] Jiménez, L, \& Vázquez1, G. A. (2005). Sequence learning under dual-task conditions: Alternatives to a resource-based account. Psychological Research, 69 (5-6), 352-368.

[5] Wierzchoń, M., Gaillard, V., Asanowicz, D., \& Cleeremans, A. (2012). Manipulating attentional load in sequence learning through random number generation. Advances in cognitive psychology, 8 (2), 179-195.

[6] Shanks, D. R. (2003). Attention and awareness in implicit sequence learning. In Jiménez, L. Attention and implicit learning. Amsterdam: Benjamins, 11-42.

[7] Shanks, D. R., Rowland, L. A., \& Ranger, M. S. (2005). Attentional load and implicit sequence learning. Psychological Research, 69 (5-6), 369-382.

[8] Lu, Z. L., Lv, Y., \& Bai, X. J. (2011). Attention load has no effect on implicit sequence learning: an eye movement study. Studies of psychology and behavior, 9 (3), 214-218.

[9] Lu, Z. L., Lv, Y., \& Shen, D. L. (2011). Attention load has no effect on implicit sequence learning: evidence from event-related potential studies. Psychological Development and Education, 27 (6), 561-568.

[10] Zhanglong Lu, Jieqiong Lin, Xiaoyu Li. An Experimental Study on Relationship Between Subliminal Emotion and Implicit Sequence Learning: Evidence From Eye Movements. International Journal of Psychological and Brain Sciences. 2018, 3 (1), 1-6.

[11] Reber. A. S. (1993). Implicit learning and tacit knowledge: An essay on the cognitive unconscious. New York: Oxford University Press.

[12] Jiménez, L. (2003). Intention, attention, and consciousness in probabilistic sequence learning. In L. Jiménez (Eds.), Attention and implicit learning (pp. 43-68). Amsterdam: Benjamins.

[13] Mayr, U. (1996). Spatial attention and implicit sequence learning: Evidence for independent learning of spatial and nonspatial sequences. Journal of Experimental Psychology: Learning, Memory, \& Cognition, 22, 350-364.

[14] Jiménez, L., \& Méndez, C. (1999). Which attention is needed for implicit sequence learning? Journal of Experimental Psychology: Learning, Memory, \& Cognition, 25, 236-259.

[15] Cock, J. J., Berry, D. C., \& Buchner, A. (2002). Negative priming and sequence learning. European Journal of Cognitive Psychology, 14, 27-48.

[16] Röttger, E., Haider, H., Zhao, F., \& Gaschler, R. (2017). Implicit sequence learning despite multitasking: the role of across-task predictability. Psychological Research. 5, 1-18.

[17] Sloutsky, V. M., \& Robinson, C. W. (2013). Redundancy matters: Flexible learning of multiple contingencies in infants. Cognition, 126, 156-164.

[18] Sævland, W., \& Norman, E. (2016). Studying Different Tasks of Implicit Learning across Multiple Test Sessions Conducted on the Web, Frontiers in Psychology, 7, 808. doi:10.3389/ fpsyg. 2016.00808. 
[19] Rowland, L. A., \& Shanks, D. R. (2006). Attention modulates the learning of multiple contingencies. Psychonomic Bulletin \& Review, 13, 634-648.

[20] Destrebecqz, A., \& Cleeremans, A. (2001). Can sequence learning be implicit? New evidence with the Process Dissociation Procedure. Psychonomic Bulletin \& Review, 8, 343-350.

[21] Jacoby, L. L. (1991). A process dissociation framework: separating automatic from intentional uses of memory. Journal of Memory \& Language, 30, 513-541.

[22] Higuchi, Y., \& Saiki, J. (2017). Implicit learning of spatial configuration occurs without eye movement. Japanese Psychological Research, 59 (2), 122-132.
[23] Kunar, M. A., Flusberg, S., Horowitz, T. S., \& Wolfe, J. M. (2007). Does contextual cuing guide the deployment of attention? Journal of Experimental Psychology: Human Perception and Performance, 33, 816-828.

[24] Schmidtke, V., \& Heuer, H. (1997). Task integration as a factor in secondary-task effects on sequence learning. Psychological Research, 60 (1-2), 53-71.

[25] Lavie, N. Distracted and confused? Selective attention under load. (2005). Trends in Cognitive Sciences, 9, 75-82.

[26] Schumacher, E. H., \& Schwarb, H. (2009). Parallel response selection disrupts sequence learning under dual-task conditions Journal of Experimental Psychology: General, 138 (2), 270-290. 